\title{
Le problème de l'analyse élémentaire quantitative des produits de pulvérisation
}

\author{
Guy Blaise \\ Laboratoire de Physique des Solides, Bâtiment 510, Université Paris-Sud, 91405 Orsay Cedex, France
}

(Received March 13, 1992; accepted May 22, 1992)

\begin{abstract}
Résumé. - La méthode de spectrométrie de masse par thermalisation des produits de pulvérisation consiste à collecter une partie de la matière pulvérisée par bombardement ionique dans une cellule en tantale portée à une température de $3000 \mathrm{~K}$. Dans cette cellule les agrégats sont dissociés et les atomes ionisés selon un processus thermique décrit par la loi de Saha-Langmuir. Un dispositif d'ionisation par impact électronique placé à l'intérieur de la cellule permet de détecter les éléments qui sont très peu ionisés par la voie thermique. La composition d'un solide est déterminée à partir des intensités mesurées, après calibrage des coefficients d'ionisation. Il est démontré que la méthode permet une analyse quantitative absolue. Des applications à l'étude des diagrammes de phases intermétalliques en couches minces, a l'analyse de microparticules, à l'étude de cinétiques réactionnelles à haute température et à celle de phénomènes de pulvérisation, sont présentées.
\end{abstract}

\begin{abstract}
Species sputtered from a solid by ion bombardment are partly collected into a tantalum cell held at $3000 \mathrm{~K}$ where they are dissociated and ionized according to the Saha-Langmuir equation. A filament stretched through the cell and negatively biased with respect to the cell wall allows the atoms to be ionized by electron impact when the thermal process becomes inefficient. Ions produced either by the thermal process or electron impact are extracted from the cell and mass analyzed. The composition of the solid is determined from ion intensities after calibration of ionization coefficients. If has been demonstrated that the method has an absolute quantitative character. Applications to elemental quantitative analysis, high temperature studies of absorbat-surface chemical reactions and studies of sputtering phenomena are being presented.
\end{abstract}

\section{Introduction.}

La matière prélevée par pulvérisation ionique à un échantillon solide se compose d'atomes et d'agrégats émis sous forme neutre plus ou moins excitée et sous forme ionisée. Les abondances des différentes espèces relèvent de mécanismes fort complexes d'échanges électroniques qui se produisent au moment où les atomes sont arrachés de la surface. Comme ces mécanismes dépendent de la nature du solide pulvérisé (composition, état chimique, ...) la proportion d'une espèce considérée n'est pas sous la seule dépendance de sa concentration. Par conséquent, toute méthode d'analyse qui sélectionne l'une des composantes de la matière pulvérisée, une 
espèce excitée ou ionisée par exemple, s'expose immanquablement à des effets de matrice, c'està-dire à des effets non linéaires en concentration, qui tiennent à la nature du solide. Mais la composition globale de la matière pulvérisée est identique à celle du matériau, une fois établi le changement de composition de surface qui assure précisément cette congruence. Une analyse quantitative absolue requiert donc de collecter sans discrimination, sinon toute la matière pulvérisée, du moins une fraction représentative de celle-ci et de disposer d'un moyen d'ionisation indépendant du mode de pulvérisation, dont le rendement est spécifique de chaque élément chimique. Mais la présence d'un abondant spectre d'agrégats dans la matière pulvérisée oblige en outre à disposer d'un moyen d'“atomisation" efficace. On voit que les conditions d'une analyse élémentaire quantitative sont sévères. La voie suggérée par Castaing en 1974 [1-3] pour y parvenir consiste à thermaliser dans une enceinte portée à haute température $(3000 \mathrm{~K})$ les produits de pulvérisation de manière à assurer un maximum de dissociation des agrégats et à tirer profit de cette thermalisation pour ioniser les éléments d'une manière spécifique.

Par la suite, se sont développées de nombreuses autres méthodes, qualifiées de méthodes de post-ionisation, qui respectent plus ou moins les principes de l'analyse quantitative énoncés [4-7]: on peut citer par exemple les méthodes dans lesquelles les produits de pulvérisation sont postionisés par laser ou par électrons [5-7], mais sans dissociation des agrégats et en éliminant les ions secondaires ou encore les méthodes qui utilisent un plasma pour "traiter" et ioniser la matière pulvérisée [4].

\section{Principe de la spectrométrie de masse des produits de pulvérisation thermalisés.}

Une partie des produits arrachés par pulvérisation au solide à analyser est collectée à travers un orifice $O$ ' et sous un angle solide $\Delta \Omega$ dans une enceinte (four) portée à une température de $3000 \mathrm{~K}$ environ (Fig. 1). Au cours de leurs multiples collisions sur les parois, les particules momentanément piégées à l'intérieur du four sont progressivement thermalisées, les agrégats sont dissociés et une proportion bien définie d'atomes se trouve ionisée. Les ions formés sont extraits de l'enceinte à travers un second orifice $O$, puis ils sont dirigés vers un spectromètre de masse multidétection pour y être analysés (Fig. 2). Les orifices $O$ ' et $O$ sont décalés de façon que les ions secondaires formés au cours de la pulvérisation ne puissent passer directement dans le spectromètre de masse.

La nécessité d'opérer à très haute température limite le choix du matériau du four à trois métaux : tantale, tungstène et rhénium. C'est le tantale qui a été choisi pour trois raisons principales : c'est le plus pur des trois en feuille mince, c'est celui qui réagit chimiquement le moins avec les atomes que l'on injecte dans le four et enfin c'est un métal qui supporte très bien de nombreux cycles thermiques sans rupture.

Pour ne pas favoriser la collecte d'une espèce par rapport à l'autre, échantillon et cellule sont portés au même potentiel. Mais cela ne préserve pas d'une discrimination qui serait due à des différences de distributions angulaires des espèces pulvérisées. Fort heureusement l'expérience montre que, dans la plupart des cas, ces différences restent faibles. Considérons un solide amorphe bombardé par un faisceau d'ions de quelques keV d'énergie, sous un angle d'incidence $\theta$ (Fig. 1). Si $S(\theta)$ est le rendement de pulvérisation du solide, le nombre $n$ de particules collectées dans la cellule chaque seconde, dans la direction $\alpha$ et sous l'angle solide $\Delta \Omega$, est :

$$
n=N_{\mathrm{p}} \frac{S(\theta)}{\pi} \Delta \Omega \cos \alpha
$$

où $N_{\mathrm{p}}$ est le nombre d'ions primaires arrivant par seonde sur l'échantillon. Dans les conditions expérimentales suivantes : ions argons de quelques keV d'énergie et de quelques $\mu \mathrm{A}$ d'intensité, 


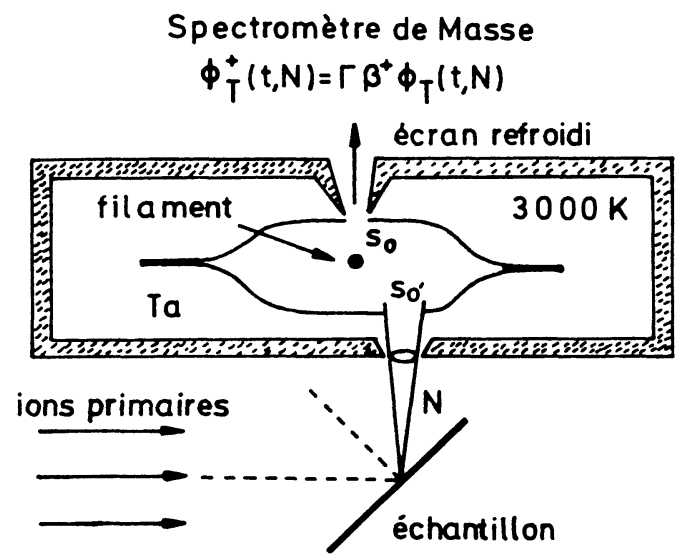

Fig. 1. - Principe de la méthode de spectrométrie de masse des produits de pulvérisation thermalisés. La cellule de tantale de $8 \mathrm{~mm}$ de diamètre et $5 \mathrm{~mm}$ de hauteur est formée de deux cupules accolées. La puissance dissipée par effet Joule dans la cellule atteint $4 \mathrm{~kW}$. Un écran refroidi absorbe le rayonnement et évite la collecte dans le spectromètre de masse d'ions produits sur la paroi externe de la cellule. Le filament de $0,4 \mathrm{~mm}$ de diamètre est polarisé négativement par rapport à la cellule pour que les électrons qu'il émet produisent une ionisation des atomes, à l'aplomb de l'orifice $s_{0}$. La distance de l'échantillon à la cellule est de $17 \mathrm{~mm}$ et l'angle solide de collecte des produits de pulvérisation est de $2 \times 10^{-3} \mathrm{str}$. Un faisceau d'ions argon de $2 \mathrm{keV}$ d'énergie et de $12 \mu \mathrm{A}$ d'intensité bombarde l'échantillon sous une incidence $\theta=45^{\circ}$. La surface utile à l'analyse étant de l'ordre du $\mathrm{mm}^{2}$ le faisceau est balayé sur une aire de $2 \times 3 \mathrm{~mm}^{2}$ de façon à assurer une érosion bien uniforme sur la partie analysée.

[Principle of the mass spectrometry method based on thermal ionization of sputtered species. Dimensions of the cell are : $5 \mathrm{~mm}$ height and $8 \mathrm{~mm}$ in diameter. The electric power dissipated in the cell by Joule effect can reach $4 \mathrm{~kW}$. Radiation emitted by the cell is absorbed by a cooled shield. The shield is also used to prevent ions produced on the external face of the cell to be collected in the mass spectrometer. Diameter of the filament is $0.4 \mathrm{~mm}$. The filament facing opening $s_{0}$ is negatively biased with respect to the cell wall. The distance from the sample to the cell is $17 \mathrm{~mm}$ and the collecting solid angle is $2 \times 10^{-3} \mathrm{str}$. The sample is bombarded under $\theta=45^{\circ}$ by an $\mathrm{Ar}^{+}$ion beam of $2 \mathrm{keV}$ energy and $12 \mu \mathrm{A}$ intensity. The beam is scanned over $2 \times 3 \mathrm{~mm}^{2}$ area in order to obtain a uniform erosion.]

$S(\theta)$ de l'ordre de quelques unités, $\alpha=45^{\circ}$ et $\Delta \Omega \approx 2 \times 10^{-3}$ srd, on trouve $n \approx 10^{11}$ atomes/s. Si l'échantillon contient un élément $\mathrm{A}$ à la concentration $C_{\mathrm{A}}$, le nombre $n_{\mathrm{A}}$ d'atomes $\mathrm{A}$ entrant dans le four chaque seconde sera :

$$
n_{\mathrm{A}}=n C_{\mathrm{A}}
$$

Le premier rôle de la cellule est d'assurer la thermalisation de la matière pulvérisée. Si $d$ est le diamètre des ouvertures $\mathrm{O}$ et $\mathrm{O}$ ' et $D$ le diamètre de la cellule supposée sphérique, le nombre moyen de collisions d'une particule sur la paroi sera $2(D / d)^{2}$ avant qu'elle ne sorte. Pour $D \simeq 10 \mathrm{~mm}$ et $d \simeq 1 \mathrm{~mm}$, cela représente plus d'une centaine de chocs, ce qui paraît amplement suffisant pour une bonne thermalisation.

Lorsque le régime permanent est atteint à l'intérieur de la cellule, c'est-à-dire qu'il entre, chaque seconde, autant de particules qu'il en sort, la densité $n_{\mathrm{g}}$ de la phase volumique est :

$$
n_{\mathrm{g}}=\frac{n}{s_{\mathrm{o}}+s_{\mathrm{o}^{\prime}}}\left(\frac{2 \pi m}{k T}\right)^{1 / 2}
$$

expression dans laquelle $s_{\mathrm{o}}$ et $s_{\mathrm{o}^{\prime}}$ sont les orifices indiqués sur la figure $1, m$ est la masse des atomes et $k$ la constante de Boltzmann. A $3000 \mathrm{~K}$ et dans les conditions expérimentales définies 


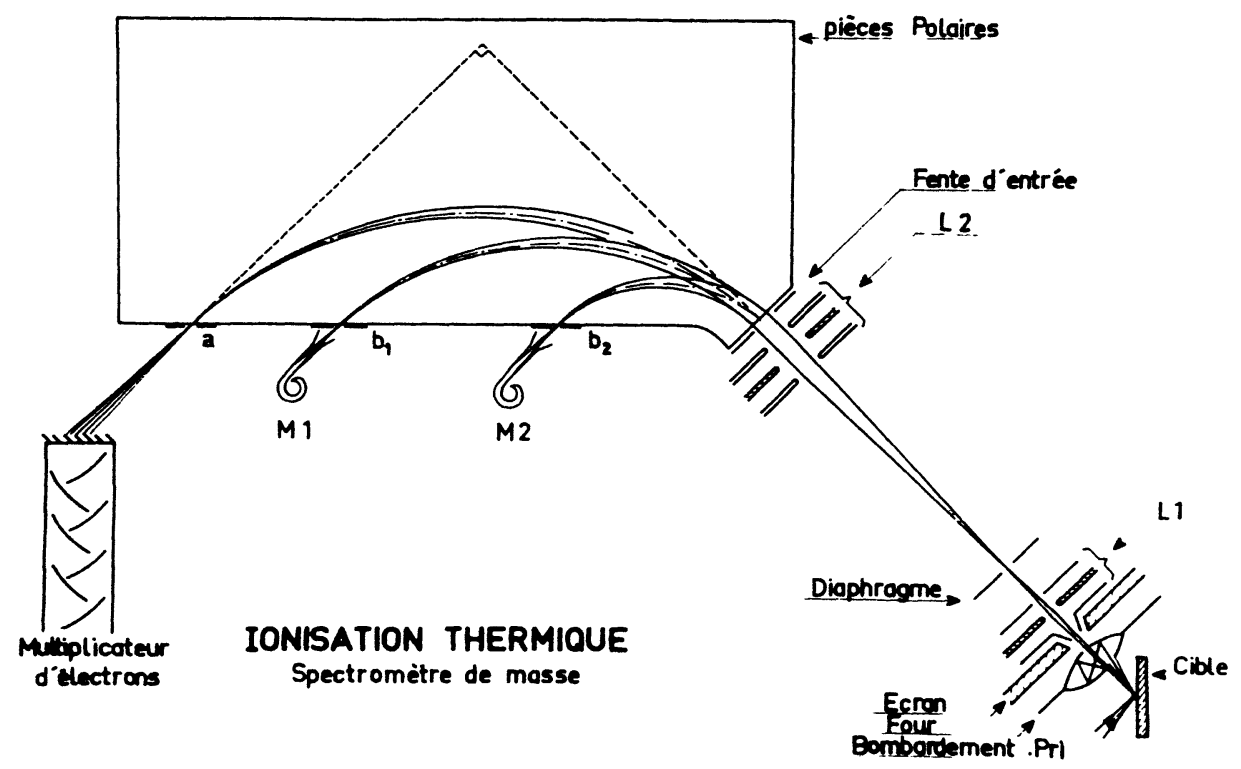

Fig. 2. - Spectromètre de masse multidétection. Les multiplicateurs d'électrons $\mathbf{M}_{1}$ et $\mathbf{M}_{2}$ sont mobiles le long de la face de sortie de l'aimant. Le multiplicateur à dinodes est à une position fixée. Il est utilisé pour mesurer le courant très intense d'ion $\mathrm{Ta}^{+}$venant de l'évaporation des parois de la cellule qui permet de régler la température de celle-ci.

[Schematic view of the multidetection mass spectrometer. Channeltrons $\mathbf{M}_{1}$ and $\mathbf{M}_{2}$ are moving along the magnet. The dinode electron multiplier is at a fixed position. It is used for measuring very intense currents like the thermal ionization current of the cell wall which allows the control of the temperature.]

précédemment, $n_{\mathrm{g}} \approx 2 \times 10^{8}$ at $/ \mathrm{cm}^{3}$ soit une pression équivalente $P_{\mathrm{g}} \approx 10^{-7}$ torr. Cette pression est bien plus faible que la pression de vapeur du tantale qui est de l'ordre de $10^{-4}$ torr vers $3000 \mathrm{~K}$

Si $\tau$ est le temps de résidence d'une particule sur la paroi de la cellule, la densité $n_{\text {ad }}$ de la phase adsorbée est :

$$
n_{\mathrm{ad}}=\tau \frac{n}{s_{\mathrm{o}}+s_{\mathbf{o}^{\prime}}}
$$

En prenant $n \approx 10^{11}$ atomes $/ \mathrm{s}, s_{\mathrm{o}} \sim s_{\mathrm{o}^{\prime}} \sim 1 \mathrm{~mm}^{2}$ et un temps moyen de collage $\tau \approx 10^{-3} \mathrm{~s}$, $n_{\mathrm{ad}} \approx 5 \times 10^{9} \mathrm{at} / \mathrm{cm}^{2}$. C'est très faible comparé à la densité surfacique des atomes de tantale qui est de $310^{14} \mathrm{at} / \mathrm{cm}^{2}$.

Ces estimations montrent d'une part que l'évolution vers l'équilibre thermique des produits de pulvérisation se fera essentiellement par des collisions sur les parois et d'autre part que la densité surfacique de la matière pulvérisée sur la paroi sera généralement bien inférieure à la monocouche.

En supposant que l'équilibre thermique soit réalisé, le degré de dissociation moléculaire à $3000 \mathrm{~K}$ atteint $10^{7}$ pour des molécules dont l'énergie de liaison est de l'ordre de $5 \mathrm{eV}$ et tombe à 0,3 lorsque cette énergie de liaison atteint $10 \mathrm{eV}$. Autrement dit, la plupart des molécules seront dissociées à l'exception d'un petit nombre d'entre elles dont l'énergie de liaison est particulièrement élevée.

La seconde fonction de la cellule est l'ionisation. A l'équilibre thermique, le coefficient d'ionisation $\alpha_{i}^{+}$d'une espèce $i$ dont le potentiel d'ionisation est $V_{i}$ est donné par l'expression 
de Saha-Langmuir :

$$
\alpha_{i}^{+} \sim \exp -\frac{e\left(V_{i}-\phi\right)}{k T}
$$

où $\phi$ est le travail de sortie de la paroi de la cellule. Avec un travail de sortie $\phi=4,10 \mathrm{~V}$ pour le tantale et pour un élément dont $V_{i}=9 \mathrm{~V}$ l'expression (5) donne $\alpha_{i}^{+} \approx 3 \times 10^{-9}$. D'après la valeur de $n$ estimée précédemment ( $n \approx 10^{11}$ atomes/s), cela conduit à un signal de l'ordre de $300 \mathrm{c} / \mathrm{s}$ pour un corps pur. Autrement dit les éléments détectables seront ceux dont le potentiel d'ionisation reste inférieur à $9 \mathrm{~V}$, c'est-à-dire essentiellement les éléments métalliques. C'est une limitation sévère pour l'exploitation analytique de la méthode. Avant de voir comment remédier à cette limitation, signalons que l'application de la relation (5) à l'ionisation des atomes de $\mathrm{Ta}$ évaporés des parois permet de réaliser un thermomètre interne dont la sensibilité est de quelques degrés vers $3000 \mathrm{~K}$. L'expression qui relie le signal tantale à la température est :

$$
I\left(\mathrm{Ta}^{+}\right)=1,7 \times 10^{13} T^{-1} \exp -\frac{135652}{T}
$$

La limitation imposée par le processus d'ionisation thermique à la détection des éléments oblige a utiliser un processus d'ionisation complémentaire qui soit compatible avec la fonction de thermalisation de la cellule. La solution adoptée consiste à ioniser par impact électronique les atomes à l'intérieur de la cellule. Pour cela on tend en travers de la cellule un filament qui fait face à l'orifice de sortie $O$ (Fig. 1) [8]. Quand le filament est polarisé négativement par rapport à la cellule, les électrons qu'il émet sont accélérés et viennent ioniser les atomes qui se trouvent au voisinage de l'orifice $O$. L'efficacité de ce processus d'ionisation est montrée figure 3 où l'on a porté le signal $\mathrm{Cu}^{+}$en fonction du potentiel négatif du filament. Lorsque $V=0$ on a le signal de thermo-ionisation du cuivre qui est de l'ordre de $1500 \mathrm{c} / \mathrm{s}$. Quand $V$ devient légèrement négatif ce signal disparaît jusqu'à ce que l'ionisation par impact d'électrons devienne efficace à partir d'une dizaine de $\mathrm{V}$. Pour $V=-50 \mathrm{~V}$ le signal cuivre atteint $16000 \mathrm{c} / \mathrm{s}$ ce qui représente un gain appréciable par rapport à la thermo-ionisation.

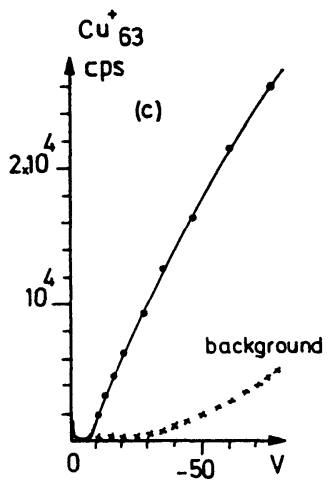

Fig. 3. - Variation du signal $\mathrm{Cu}_{63}^{+}$et du bruit de fond en fonction de la tension appliquée sur le filament de la cellule.

[Variation of $\mathrm{Cu}_{63}^{+}$signal and background as a function of the biased voltage on the filament.]

Comme l'efficacité de l'ionisation par impact d'électrons ne dépend pas trop fortement du potentiel d'ionisation, tous les éléments sont en principe détectables avec des rendements du 
même ordre de grandeur. Cet effet est particulièrement bien mis en évidence sur la figure 4 où sont comparés les signaux $\mathrm{Ga}^{+}, \mathrm{As}^{+} \mathrm{et} \mathrm{Sb}^{+}$en thermoionisation et en ionisation par impact d'électrons. En thermo-ionisation l'arsenic et l'antimoine n'apparaissent pas en raison de leurs potentiels d'ionisation trop élevés. Avec l'ionisation par impact d'électrons les trois éléments présentent des signaux comparables. Mais en contrepartie, des pics parasites apparaissent.
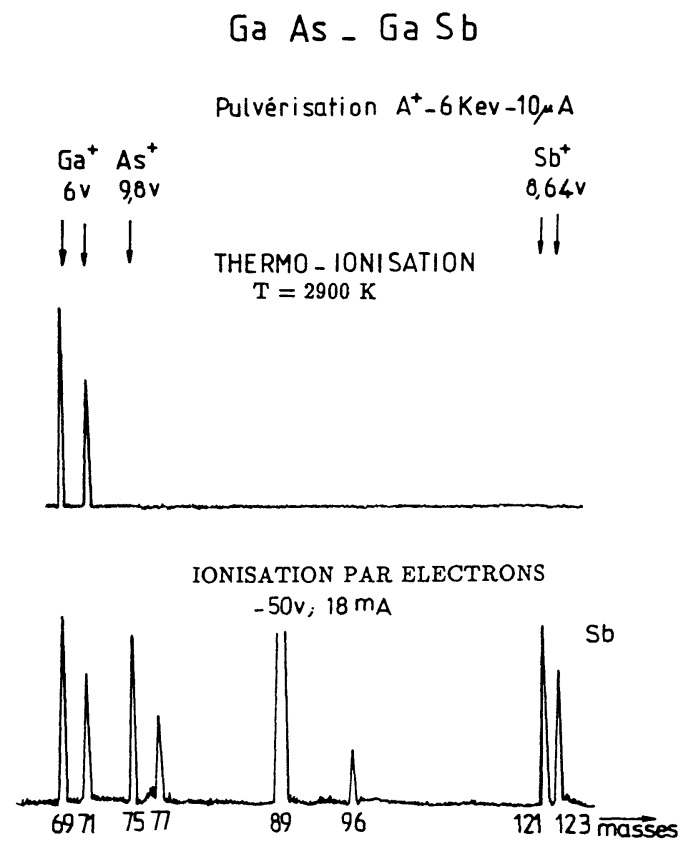

Fig. 4. - Intensités des ions $\mathrm{Ga}$, As et $\mathrm{Sb}$ mesurées en thermo-ionisation et en ionisation par impact d'électrons. Les éléments As et $\mathrm{Sb}$ ne sont pas détectables en thermo-ionisation en raison du potentiel d'ionisation trop élevé de ces éléments. Les rendements d'ionisation par impact d'électrons sont à peu près les mêmes pour les trois éléments. Des pics parasites aux masses 89 et 96 apparaissent.

[Intensities of $\mathrm{Ga}$, As and $\mathrm{Sb}$ ions produced by the thermal process or by electron impact. The ionization potentials of $\mathrm{As}$ and $\mathrm{Sb}$ are too high to make these elements detectable using the thermal ionization process. Ionization yields obtained by electron impact are about the same for the three elements. Spurious peaks appear at masses 89 and 96 .]

Avec le système d'ionisation par impact d'électrons, le rendement utile incluant la transmission du spectromètre de masse et le rendement du détecteur est de $10^{-6}$ environ. Cela conduit à des signaux de l'ordre de $10^{5} \mathrm{c} / \mathrm{s}$ pour des corps purs.

\section{Procédure d'analyse quantitative.}

Quel que soit le mode d'ionisation choisi, thermique ou par impact électronique, le rapport des intensités de deux éléments $\mathrm{A}$ et $\mathrm{B}$ de concentrations respectives $C_{\mathrm{A}}$ et $C_{\mathrm{B}}$ s'exprime par :

$$
\frac{I\left(\mathrm{~A}^{+}\right)}{I\left(\mathrm{~B}^{+}\right)}=k_{\mathrm{AB}} \frac{C_{\mathrm{A}}}{C_{\mathrm{B}}}
$$


où $k_{\mathrm{AB}}$ est le coefficient relatif d'ionisation.

Le caractère quantitatif absolu de la méthode a été vérifié en montrant que $k_{\mathrm{AB}}$ est caractéristique des éléments $\mathrm{A}$ et $\mathrm{B}$ et indépendant du composé dans lequel ils se trouvent. La méthode utilisée consiste à mesurer $k_{\mathrm{AB}}$ sur une séquence fermée de composés tels que A-B, B-C, ... X-A. Si l'analyse est quantitative on doit trouver :

$$
k_{\mathrm{AB}} \cdot k_{\mathrm{BC}} \ldots k_{\mathrm{XA}}=1
$$

Dans les deux exemples présentés dans le tableau I, on trouve 1,07 et 0,98 en utilisant respectivement l'ionisation thermique et l'ionisation par impact d'électrons.

Tableau I.

\begin{tabular}{|l|c|c|c|c|c|c|}
\hline & $\mathrm{Al}-\mathrm{Cu}$ & $\mathrm{Cu}-\mathrm{Mg}$ & $\mathrm{Mg}-\mathrm{Al}$ & $\mathrm{Al}-\mathrm{Zn}$ & $\mathrm{Zn}-\mathrm{Cu}$ & $\Pi$ I $k_{\mathrm{AB}}$ \\
\hline$k_{\mathrm{AB}}^{\text {th }}$ & 468 & 0,091 & 0,025 & & & 1,07 \\
$k_{\mathrm{AB}}^{\mathrm{e}}$ & & 1,25 & 0,205 & 2,2 & 1,75 & 0,98 \\
\hline
\end{tabular}

Ces résultats sont compatibles avec la valeur théorique compte tenu d'une précision de 5\% sur la mesure de chaque coefficient. Le fait de vérifier le caractère quantitatif absolu de l'analyse dans le cas de l'ionisation par impact d'électrons est très important, car on pouvait craindre que le champ électrique produit dans la cellule par le filament soit préjudiciable à cette analyse.

D'un point de vue pratique $k_{\mathrm{AB}}$ peut être mesuré à partir de n'importe quel standard qui contient des éléments $\mathrm{A}$ et $\mathrm{B}$ à des concentrations connues. Il n'est pas nécessaire d'utiliser des standards de composition proche de celle de l'échantillon, comme c'est le cas en SIMS par exemple. La limite de détection calculée pour une analyse de surface en pulvérisant une ou deux couches atomiques de matière sur une aire de $1 \mathrm{~mm}^{2}$ environ est de $0,1 \%$ avec une précision de $10 \%$ lorsqu'on utilise l'ionisation par impact d'électrons. En fait deux limitations à la détection sont à prendre en compte pour certains éléments, en dehors des problèmes de métrologie. La première est liée à la réactivité chimique de la paroi tantale. L'énergie d'adsorption de certains éléments est si élevée que des effets mémoires apparaissent (exemple le niobium) tandis que d'autres tels que l'oxygene, le carbone, le bore, le platine... se dissolvent dans la paroi de tantale de la cellule, ce qui les rend indétectables. La seconde limitation vient des composants de l'atmosphère résiduelle qui sont ionisés par impact d'électrons. Certains éléments tels que $\mathrm{N}_{2}$ par exemple donnent des signaux si intenses qu'il est impossible de détecter le silicium à la masse 28.

Toutes les améliorations que l'on pourra apporter ne permettront pas d'assurer une analyse latérale de l'échantillon à l'échelle du $\mu \mathrm{m}$ comme c'est le cas en SIMS. Par contre, l'analyse en profondeur est possible dans des conditions de résolution aussi bonnes qu'en SIMS. Dans le cas présent l'analyse en profondeur est significative si l'échantillon est de composition homogène sur une aire de quelques $\mathrm{mm}^{2}$. Une érosion uniforme est obtenue en balayant le faisceau de bombardement sur ces quelques $\mathrm{mm}^{2}$. Pour des ions primaires $\mathrm{Ar}^{+}$d'énergie comprise entre 2 et $5 \mathrm{keV}$ et de densité de courant d'environ $2 \mu \mathrm{A} / \mathrm{mm}^{2}$, l'érosion progresse à une vitesse de quelques couches atomiques par seconde. Comme les signaux sont intégrés sur une seconde, chaque intégration représente la composition moyenne de l'échantillon sur ces quelques couches atomiques.

Dans la plupart des méthodes d'analyse en profondeur, les intensités sont portées en fonction du temps d'érosion. La calibration en profondeur est ensuite obtenue en mesurant la profondeur du cratère. Cette procédure requiert des conditions de pulvérisation très stables, de même qu'une 
vitesse d'érosion bien constante. Sauf en ce qui concerne les semi-conducteurs, cette dernière condition n'est pratiquement jamais remplie.

Le fait que l'analyse soit quantitative permet ici d'adapter la procédure employée en microanalyse par sonde électronique [9], à savoir de porter les concentrations (atomiques ou en masse) en fonction de la masse pulvérisée par unité de surface. Dans la plupart des cas il n'est pas nécessaire de convertir en profondeur cette masse par unité de surface pour saisir tout l'intérêt du profil relevé. Cette procédure a l'avantage de donner des profils qui sont indépendants des conditions de pulvérisation, d'autant plus que les signaux sont détectés simultanément dans le spectromètre de masse (Fig. 2). Les fluctuations d'intensité ou d'énergie du canon n'ont aucune incidence sur la forme du profil.

\section{Applications de la méthode.}

4.1 DiAgRAMMES DE PHASES PAR INTERDIFFUSION MÉTALLIQUE EN COUCHES MINCES [10-11]. - Le principe consiste a déposer une couche mince $(\sim 100 \mathrm{~nm})$ sur un substrat dont la surface a été soigneusement polie et nettoyée. Après recuit à une température assez basse (200 à $500^{\circ} \mathrm{C}$ selon les systèmes) pendant un temps court (quelques heures) on procède à l'analyse en profondeur sur des épaisseurs de quelques centaines de $\mathrm{nm}$. Un exemple est donné figure 5 pour le couple Al-Ni. Après quelques heures de recuit on observe une série de plateaux qui correspondent à des composés définis $\left(\mathrm{Al}_{3} \mathrm{Ni}\right.$ ou $\left.\mathrm{AlNi}_{3}\right)$ du diagramme d'équilibre thermodynamique ou qui se forment aux limites de phases de ce diagramme. L'apparision de composés aux limites de phase s'explique par un coefficient de diffusion plus grand sur ces limites qu'à la composition stoechiométrique des phases $\left(\mathrm{Al}_{3} \mathrm{Ni}_{2}\right.$ ou $\mathrm{AlNi}$ par exemple). Ces études sur les diagrammes de phase en couches minces ont révélé des écarts de composition à la germination, ont permis de préciser les limites de phases, de suivre la cinétique de croissance des composés et de mesurer les coefficients de diffusion chimique dont les valeurs très élevées sont de quelques $10^{-16} \mathrm{~cm}^{2} / \mathrm{s}$. Actuellement on utilise la méthode pour caractériser les interfaces dans tous les problèmes de dépôt et de traitement thermique des couches minces.

4.2 ANALYSE DE MICROPARTICULES. - R. Castaing a suggéré d'utiliser la méthode pour l'analyse de microparticules dont la taille est comprise entre 0,1 et $10 \mu \mathrm{m}$. Dans l'exemple traité, des particules de composition $\left(\mathrm{Al}_{2} \mathrm{O}_{3}\right)_{0,1}-\left(\mathrm{Cr}_{2} \mathrm{O}_{3}\right)_{0,9}$ pré parées par pressage à chaud, sont étalées sur une grille de $8 \mu \mathrm{m}$ de pas et $30 \%$ de transparence, placée au-dessus d'un petit orifice pratiqué dans la cellule. De légers chocs sur la tige qui fixe la grille à la paroi de l'appareil permettent de faire tomber, par gravitation, des grains dans la cellule. L'histogramme de la figure 6 représente le nombre de grains analysés en fonction du rapport des signaux $n\left(\mathrm{Cr}^{+}\right) / n\left(\mathrm{Al}^{+}\right)$. Un pic dont le maximum se situe à 0,108 , c'est-à-dire tout près de la valeur standard 0,105 , est observé. La dispersion de l'ord re de $10 \%$ s'explique par la fluctuation statistique du signal chrome peu intense dans les petits grains. Une saturation du détecteur du signal aluminium très intense dans les gros grains explique la "traînée" observée à droite du pic.

Une olivine de composition $\mathrm{SiO}_{4} \mathrm{Mg}_{x} \mathrm{Fe}_{y} \mathrm{Ca}_{0,02}$ a été étudiée dans les mêmes conditions que précédemment. De la position du pic observé pour le rapport des signaux $\mathrm{Fe}^{+} / \mathrm{Mg}^{+}$on a déduit un rapport $x / y=0,12 \pm 0,02$. L'étude à la sonde électronique de cette olivine a donné un résultat tout à fait comparable : $x / y=0,113 \pm 0,004$.

Ces exemples montrent l'intérêt de la méthode pour l'analyse de grains de poussières. Mais il ne faut pas sous-estimer les problèmes spécifiques que cela pose :

i) contamination de la cellule par le film liquide qui se forme lors de la fusion d'un grain (effets mémoire pour certains éléments); 

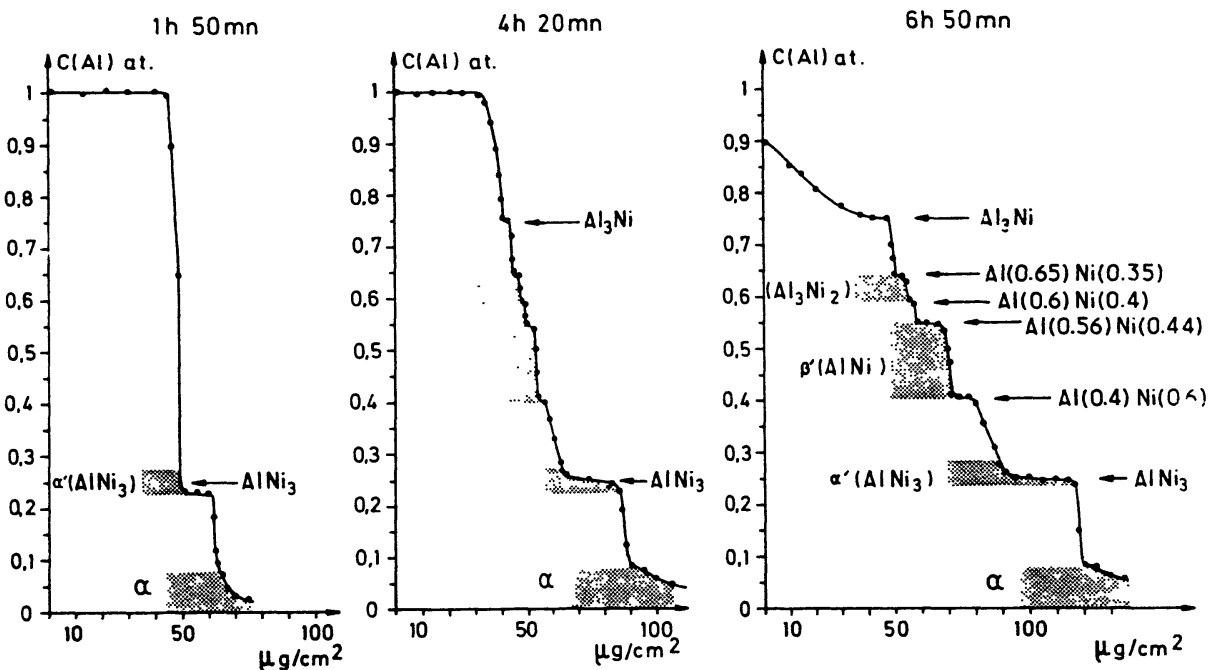

Fig. 5. - Profils de concentration d'aluminium en fonction de la masse par unité de surface érodée dans le système Al-Ni. La température de diffusion était de $220^{\circ}$ : (a) après $1 \mathrm{~h} 50$ de diffusion, (b) $4 \mathrm{~h} 20$ et (c) $6 \mathrm{~h} 50$. [Al-Ni diffusion diagram obtained at $220^{\circ} \mathrm{C}$. Aluminium concentration profile plotted versus mass thickness : (a) after $1 \mathrm{~h} 50 \mathrm{mn}$ diffusion ; (b) after $4 \mathrm{~h} 20 \mathrm{~mm}$; (c) after $6 \mathrm{~h} 50 \mathrm{mn}$.]

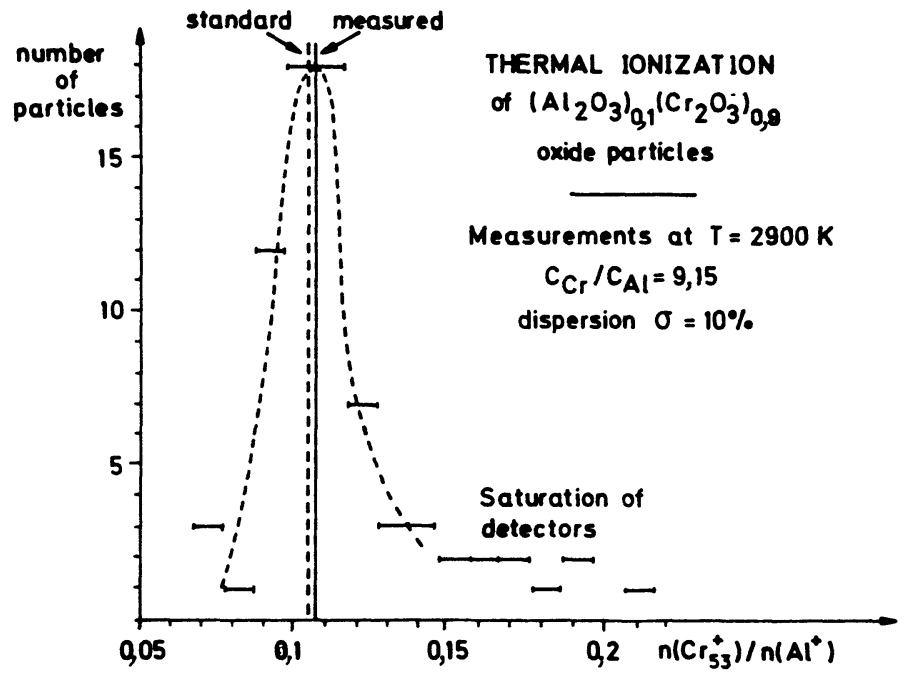

Fig. 6. - Analyse de micro-particules d'oxide $\left(\mathrm{Al}_{2} \mathrm{O}_{3}\right)_{0,1}\left(\mathrm{Cr}_{2} \mathrm{O}_{3}\right)_{0,9}$ : histogramme du nombre de particules analysées en fonction du rapport des intensités des ions $\mathrm{Cr}^{+}$et $\mathrm{Al}^{+}$. La dispersion à gauche du pic est due à des fluctuations statistiques d'intensités et à droite à la saturation des détecteurs.

$\left[\left[\mathrm{Al}_{2} \mathrm{O}_{3}\right]_{0.1}\left[\mathrm{Cr}_{2} \mathrm{O}_{3}\right]_{0.9}\right.$ oxide particle analysis. Histogram of the number of particles versus $\mathrm{Cr}^{+} / \mathrm{Al}^{+}$ intensity ratio. Deviation from the standard value of 0.105 is due to statistical fluctuations on the left of the peak and detector saturation on the right.] 
ii) distillation fractionnée de certains éléments ;

iii) saturation des détecteurs due à l'extrême intensité des signaux. Par exemple une particule de $1 \mu \mathrm{m}^{3}$ délivre des signaux de plusieurs centaines de milliers de coups en des temps inférieurs au $1 / 10$ de seconde.

4.3 ETUDE DE CINETTIQUES RÉACTIONNELLES À HAUTE TEMPÉRATURE (2000 à $3000 \mathrm{~K})$ [12]. Les atomes introduits dans la cellule peuvent soit désorber de la paroi après un temps moyen de collage, soit pénétrer dans le matériau et diffuser. En étudiant, en fonction de la température, le flux de particules qui sort de la cellule (Fig. 1), on peut suivre les réactions qui se passent à l'intérieur. L'exemple présenté figure 7 concerne le système uranium-tantale. Sur cette figure sont portés, en fonction de la température, le flux $\phi_{\mathrm{T}}^{+}$d'uranium qui sort de la cellule et le temps $\tau_{\mathrm{m}}$ nécessaire à la stabilisation de ce flux. De l'analyse de ces résultats on déduit l'énergie d'adsorption de l'uranium sur le tantale $\left(E_{\mathrm{a}}=2,4 \mathrm{eV}\right)$ et on dresse l'évolution du temps de collage en fonction de la température. En outre on met en évidence la présence d'une solution solide dont la concentration limite vers $2700 \mathrm{~K}$ est estimée à $0,3 \%$ et on suit la cinétique de diffusion dans cette phase, en fonction de la température.

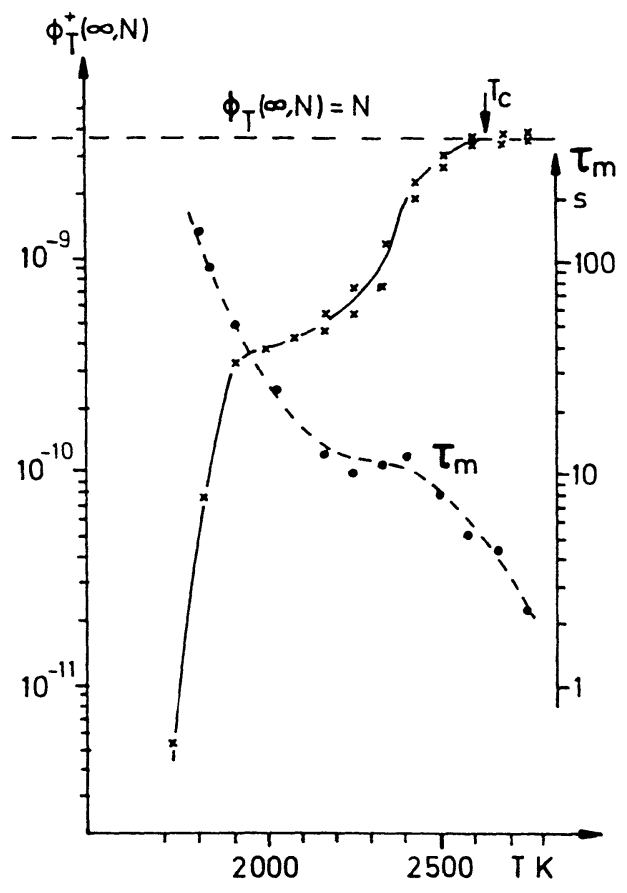

Fig. 7. - Variation en fonction de la température du flux $\phi_{T}^{+}$d'ions $U^{+}$détectés dans l'état stationnaire (courbe en trait plein) et de la constante de temps d'évolution $\tau_{\mathrm{m}}$ du flux vers l'état stationnaire (courbe en tirets).

[Flux $\phi_{\mathrm{T}}^{+}$of $\mathrm{U}^{+}$ions detected in the stationary state (full curve) and characteristic time $\tau_{\mathrm{m}}$ of the evolution of the signal to the stationary state (dashed curve), versus temperature.] 


\section{Etude des phénomènes de pulvérisation [13].}

De nombreux phénomènes liés à la pulvérisation peuvent être étudiés en changeant la nature ou l'énergie des ions de bombardement : par exemple la rugosité induite par la pulvérisation ou l'énergie de seuil de pulvérisation. L'exemple présenté figure 8 concerne l'étude du changement de composition de surface sur un alliage $\mathrm{Cu}_{0,67} \mathrm{Zn}_{0,33}$. Le suivi, en fonction du temps d'érosion, des concentrations de cuivre et de zinc dans la matière pulvérisée révèle une pulvérisation préférentielle du zinc : la concentration de zinc qui est supérieure à la valeur nominale de 0,33 au début de l'érosion décroît progressivement à mesure que la surface s'enrichit en cuivre, pour finalement atteindre la valeur nominale. Le comportement du cuivre est inverse de celui du zinc. Sur la figure 8 sont également portées les concentrations de surface relevées par spectrométrie Auger. On constate un changement de composition de surface très important. Cet exemple illustre bien le problème de l'analyse Auger quantitative associée à une érosion ionique.

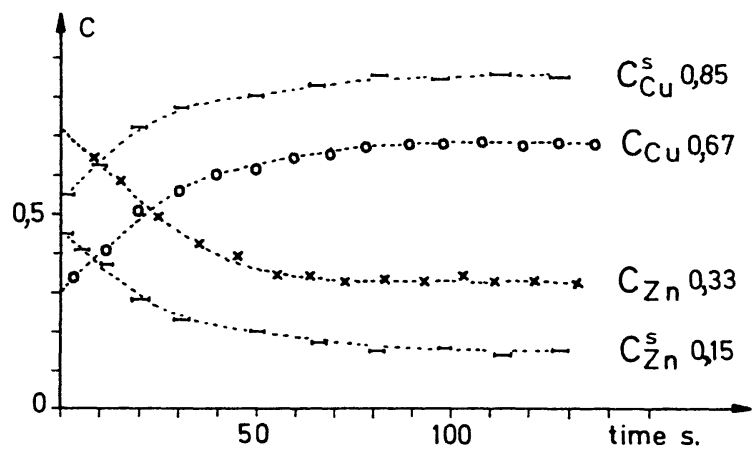

Fig. 8. - Evolution en fonction du temps des concentrations de $\mathrm{Zn}$ et de $\mathrm{Cu}$ (croix et cercles) dans la matière pulvérisée d'un alliage $\mathrm{Cu}_{0,67} \mathrm{Zn}_{0,33}$ par un faisceau d'ions $\mathrm{Ar}^{+}$de $4 \mathrm{keV}$ d'énergie. Les courbes en tirets représentent les évolutions des concentrations de surface de l'alliage, mesurées par spectrométrie Auger.

[Time evolution of $\mathrm{Zn}$ and $\mathrm{Cu}$ concentrations (crosses and open circles) in the sputtered material from a $\mathrm{Zn}_{0.33} \mathrm{Cu}_{0.67}$ alloy bombarded with $4 \mathrm{keV}$ energy $\mathrm{A}^{+}$ions. Dashed curves are surface concentrations as measured by Auger.]

\section{Conclusion.}

La méthode de spectrométrie de masse des produits de pulvérisation thermalisés s'est révélée être pleinement satisfaisante du point de vue de l'analyse quantitative des solides. En particulier on notera que le dispositif d'ionisation par électrons placé dans la cellule, qui permet d'accroître le nombre d'éléments détectables par rapport à la thermoionisation, conserve le caractère absolu de l'analyse. Les seuls éléments qui ne sont pas détectés par ce procédé sont ceux qui réagissent chimiquement avec la cellule (B, $\mathrm{C}$ et $\mathrm{O}$ notamment) et ceux dont la masse correspond à un composant majoritaire de l'atmosphère résiduelle.

La sensibilité de la méthode est comparable, voire supérieure, à celle des spectrométries d'électrons. Le domaine d'application majeur est celui de l'analyse en profondeur de couches 
minces latéralement homogènes. Dans ce domaine des résultats originaux ont été obtenus sur la diffusion multiphase d'alliages $\mathrm{Al}-\mathrm{Ni}, \mathrm{Cu}-\mathrm{Al}, \mathrm{Al}-\mathrm{Ti}$ et maintenant sur les céramiques d'oxydes supraconducteurs à haute température.

\section{Bibliographie}

[1] Castaing R. et Blaise G., X-rays and Microanalysis Conference, Boston (1977).

[2] Blaise G. et Castaing R., C.R. Acad. Sci Paris série B 281 (1977) 449.

[3] Blaise G. et Castaing R., J. Microsc. Spectrosc. Electron. 3 (1978) 439.

[4] OECHSNER H., RUHE W., STUMPE E., Surf. Sci 85 (1979) 289.

[5] GNASER H., FleischHAUER J. and HOFER W.O., Appl Phys. 38 (1985).

[6] Pellini M.J., Young C.E., CALAWAY W.F. and GRUEN D.M., Surf. Sci. 144 (1984) 619. BECKER C.H. and GILLEN K.T., Anal. Chem. 56 (1984) 1671.

[7] COUCHOURON M. and HENNEQUIN J.F., Int. J. Mass Spectrom and Ion Process 69 (1986) 1-15.

[8] Blaise G., Castaing R. et Quettier R., J. Phys. Colloq. France 45 (1984) C2-125.

[9] HEINRICH K.F.J., Electron beam X-ray microanalysis (Van Nostrand Reinhold, NY) 10 (1981) 255-301.

[10] TARENTO R.J. et Blaise G., Acta Metall. 36 (1988) 1035.

[11] TARENTO R.J. et BlaISE G., Acta Metall 37 (1989) 2305.

[12] Blaise G. et Seghrouchni E., J. Phys. I France 1 (1991) 931.

[13] BLAISE G., Interactions plasmas froids/surfaces (Les Editions de Physique, 1988) pp. 259-282. 